\title{
$M I G 7$ is involved in vasculogenic mimicry formation rendering invasion and metastasis in hepatocellular carcinoma
}

\author{
BO $\mathrm{QU}^{1 *}$, GUANNAN SHENG ${ }^{2 *}$, LONG GUO $^{3}$, FEI YU $^{2}$, GUANNAN CHEN $^{2}$, \\ QI LU ${ }^{4}$, RENJIE WANG ${ }^{5}$, BING HAN $^{3}$ and YI LU $^{3}$ \\ ${ }^{1}$ Department of Oncological Surgery, Affiliated Hospital of the Logistics University of Chinese People's Armed Police Forces, \\ Tianjin 300162; ${ }^{2}$ Department of Oncological Surgery, Postgraduate Training Basement of Jinzhou \\ Medical University in the Affiliated Hospital of the Logistics University of Chinese People's Armed Police Forces, \\ Tianjin 300162; ${ }^{3}$ Department of Hepatobiliary Surgery, The First Affiliated Hospital of Medical College, \\ Xi'an Jiaotong University, Xi'an, Shaanxi 710061; ${ }^{4}$ Institute of Disaster Medicine and Public Health, Affiliated Hospital of \\ the Logistic University of Chinese People's Armed Police Force, Tianjin 300162; ${ }^{5}$ Department of Clinical Laboratory, \\ Affiliated Hospital of the Logistics University of Chinese People's Armed Police Forces, Tianjin 300162, P.R. China
}

Received November 13, 2016; Accepted November 16, 2017

DOI: 10.3892/or.2017.6138

\begin{abstract}
Migration-inducing gene 7 (MIG7) is highly expressed and is implicated in multiple malignant tumors with vasculogenic mimicry (VM) which renders possible routes without the endothelium for invasion and metastasis. However, there are few reports in the literature describing the relationship between $M I G 7$ expression and VM formation in hepatocellular carcinoma (HCC). In the present study, we found a significantly positive correlation between $M I G 7$ expression and VM in 40 HCC specimens. Three-dimensional (3D) culture showed that VM formation in the HCC cell line MHCC-97H with high metastatic potential was enhanced to a greater extent than that of MHCC-97L and Huh-7 with low and non-metastatic potential. There was no VM formation in human normal hepatocyte line L-02. Moreover, MIG7 expression was higher in MHCC-97H than in MHCC-97L and Huh-7 cells and non-detectable in L-02 cells. MIG7 knockdown in MHCC-97H cells reduced VM formation, and weakened the invasive properties accompanying the enhanced cellular adhesion. Notably, there was no significant effect of endostatin (ES), a broad-spectrum angiogenesis inhibitor applied to clinical treatment, on both MIG7 expression and VM formation. Thus, the present study presents a causal link between $M I G 7$
\end{abstract}

Correspondence to: Dr Bo Qu, Department of Oncological Surgery, Affiliated Hospital of Logistics University of Chinese People's Armed Police Forces, 220 Chenglin Road, Hedong, Tianjin 300162, P.R. China

E-mail: doctor_qb@163.com

${ }^{*}$ Contributed equally

Key words: migration-inducing gene 7, angiogenesis, metastasis, invasion, endostatin expression and $\mathrm{VM}$ formation in $\mathrm{HCC}$, suggesting a potential treatment target for invasion and metastasis.

\section{Introduction}

Hepatocellular carcinoma (HCC) is one of the most common malignancies, and is the second leading cause of cancerrelated death worldwide (1). HCC accounts for $91 \%$ of primary liver cancer cases and presents poor prognosis with an overall 5 -year survival rate of $<5 \%$ (2). Like other malignant tumors, the metastasis and recurrence of $\mathrm{HCC}$ has become one of the major obstacles to the therapeutic treatment of HCC.

Although blood vessels are considered as a main route of HCC metastasis, vasculogenic mimicry (VM) has been certified to be a potential bypass for metastasis. VM is a microvascular channel which occurs de novo without the presence of endothelial cells (3). In VM, tumor cells arrange in lines to form vessel-like structure effectively mimicking a true vascular endothelium, which provide tumors with blood perfusion and promote tumor metastasis $(4,5)$. VM has been reported in HCC (6) and other types of tumors including ovarian cancer (7), melanoma (8), osteosarcoma (9) and prostatic cancer (10).

VM is associated with poor patient prognosis and is considered as a loophole for antiangiogenesis therapy. Currently, antiangiogenic therapy mainly targets endothelium-dependent angiogenesis. Several antiangiogenic agents such as endostatin (ES) have been proved to be effective in inhibiting endothelium-dependent angiogenesis in both mice and humans $(11,12)$. However, in the clinical trials, they failed to decrease tumor metastasis, and instead, tended to increase the risk of occurrence (13). Antiangiogenic therapy can lead to hypoxia, which contributes to the formation of VM, thus, facilitating tumor metastasis. Therefore, an effective treatment strategy should target not only endothelium-dependent vessels but also endothelium-independent vessels such as VM $(14,15)$. Recently, the effects of caspases, including caspase-3, -6 and -7 , were found to play a key role in apoptosis and may also play an important 
role in tumorigenesis, particularly in VM $(16,17)$. However, the mechanism by which VM formation is promoted remains elusive.

Overexpression of migration-inducing gene 7 (MIG7) is found in highly aggressive tumors with VM rather than nonaggressive malignant cells without VM (18-20) suggesting an important role in VM formation and cancer aggression. Recently, it was reported that $M I G 7$ was found to be related to the formation of VM in gastric cancer, and to play a complementary role in growth factors and COX-2/PGE2-related cancer invasion and metastasis $(20,21)$. In the present study, we investigated the association of MIG7 expression with VM formation in HCC and its effects on the potential for HCC invasion and metastasis.

\section{Materials and methods}

Patient samples and cell lines. Forty matched pairs of HCC paraffin-embedded specimens from 40 patients (male, $n=28$; female, $\mathrm{n}=12$; mean age, 54) and 10 normal liver paraffinembedded specimens were purchased from Shaanxi Chaoying Clinical Pathology Institute (Shaanxi, China). The use of the specimens in the study was approved by the Ethics Committee of the Affiliated Hospital of the Logistics University of Chinese People's Armed Police Forces. Human HCC cell lines (MHCC-97H, MHCC-97L, Huh-7) and human normal hepatocyte L-02 cells were purchased from the Liver Cancer Institute of Fudan University (Shanghai, China). Dulbecco's modified Eagle's medium (DMEM) with $10 \%$ fetal bovine serum (FBS) was used to culture all 4 types of cells at $37^{\circ} \mathrm{C}$ and $5 \% \mathrm{CO}_{2}$.

Primary antibodies and reagents. Rabbit anti-human MIG7 (cat. no. ab83494) was purchased from Abcam (Cambridge, MA, USA). Mouse anti-human CD34 monoclonal antibody (cat. no. sc-19621), mouse anti-human $\beta$-actin monoclonal antibody (cat. no. 130065), goat anti-rabbit IgG (cat. no. sc-2004), goat anti-mouse IgG (cat. no. sc-2005) and laminin rabbit anti-human polyclonal antibody (cat. no. sc-5582) were purchased from Santa Cruz Biotechnology (Santa Cruz, CA, USA). Fluorescence marked (FITC) goat anti-mouse IgG (cat. no. 555988) and fluorescence marked (TRITC) goat anti-mouse IgG (cat. no. 610055) were purchased from BD Biosciences (San Jose, CA, USA). High Fidelity PrimeScript ${ }^{\mathrm{TM}}$ RT-PCR kit and PrimeSTAR ${ }^{\circledR}$ HS DNA Polymerase were purchased from Takara Bio Group (Dalian, China). Matrigel and cell culture plates were purchased from Corning Inc. (Corning NY, USA). ES was purchased from Calbiochem (San Diego, CA, USA) and 0.25\% tryptase was purchased from Sigma-Aldrich (St. Louis, MO, USA).

Immunostaining analysis. For MIG7 protein detection, formalin-fixed and paraffin-embedded sections were deparaffinized and hydrated in a graded ethanol series. After antigen retrieval, sections were treated with $10 \%$ goat serum for 20 min and incubated with rabbit anti-human MIG7 polyclonal antibody $(1: 100)$ at $4^{\circ} \mathrm{C}$ overnight, and in turn biotinylated antibody and streptavidin-peroxidase at $37^{\circ} \mathrm{C}$ for $30 \mathrm{~min}$. Signaling was detected with DAB substrate for $5 \mathrm{~min}$. For VM formation detection, conventional treated sections were incubated with rabbit anti-human laminin polyclonal antibody (1:200) at $37^{\circ} \mathrm{C}$ for $30 \mathrm{~min}$, and then treated with DAB for $5 \mathrm{~min}$. The sections were then dehydrated and mounted with
Permount and viewed by bright-field microscopy. The results, statistically analyzed according to the positive staining rate of the malignant cells and staining intensity, were assessed by the independent film reading of two professional physicians in a blinded manner. The three levels of staining intensity were: negative $(-, 0)$; weakly positive $(+, 1)$; intensely positive $(++, 2)$.

$3 D$ cell culture. 3D culture was employed to detect VM formation in vitro. Six-wells of culture plates were coated with Matrigel (50 $\mu \mathrm{l} /$ well). The cells were maintained in DMEM supplemented with $10 \%$ FBS and trypsinized, and then, suspended at $1 \times 10^{6} / \mathrm{ml}$ in complete medium. Finally, the cells were seeded on gels and incubated at $37^{\circ} \mathrm{C}$ in $5 \% \mathrm{CO}_{2} / 9 \%$ air. The tube-like connections were observed under an inverted microscope. The number of tube-like connections per field (x200 magnification) was counted (22). Five random fields were analyzed in each sample.

Semi-quantitative PCR analysis. The semi-quantitative PCR was employed to detect $M I G 7$ mRNA in different cell groups and the $\beta$-actin RNA was used as the internal standard control. According to the instructions, TRIzol reagent (Invitrogen, Carlsbad, CA, USA) was used to harvest the total RNA. Subsequently, according to the manufacturer's protocol, the High Fidelity PrimeScript ${ }^{\mathrm{TM}}$ RT-PCR kit (Takara Bio) was employed to transform to cDNA with $1 \mu \mathrm{g}$ of total RNA. Then, the resulting cDNA was used for semi-quantitative PCR with SYBR-Green reagents (Fermentas, Waltham, MA, USA). The sequences of the primers were as follows: $M I G 7$ mRNA forward: 5'-TCT CAG GCA GTC AGT GGG-3' and $M I G 7$ mRNA reverse: 5'-GTT GGA TGG GAT GTC TCG-3'; $\beta$-actin mRNA forward: 5'-ATC GTG CGT GAC ATT AAG GAG AAG-3' and $\beta$-actin mRNA reverse: 5'-AGG AAG GAA GGC TGG AAG AGT G-3'. The cycling parameters were $94^{\circ} \mathrm{C}$ for $2 \mathrm{~min}$, then 40 cycles of $94^{\circ} \mathrm{C}$ for $10 \mathrm{sec}$ and $60^{\circ} \mathrm{C}$ for $30 \mathrm{sec}$, followed by a melting curve analysis. Dissociation curve analysis was applied to confirm the specificity of the PCR amplification. Relative expression levels were determined using the housekeeping gene $\beta$-actin for normalization.

Western blot assay. All cells (MHCC-97H, MHCC-97L, Huh-7 and L-02) were washed with phosphate-buffered saline (PBS) and the lysates were prepared using modified radioimmunoprecipitation assay buffer at $4^{\circ} \mathrm{C}$ for $15 \mathrm{~min}$. The proteins were resolved by $0.1 \%$ sodium dodecyl sulfate-polyacrylamide gel electrophoresis (SDS-PAGE) and transferred onto nitrocellulose filter membranes. Subsequently, blots were blocked by TBST and incubated overnight with primary antibodies (MIG7 1:200, $\beta$-actin 1:500) at $4^{\circ} \mathrm{C}$. Then, the blots were washed in TBS containing $0.1 \%$ Tween-20 and labeled with goat anti-rabbit $\operatorname{IgG-HRP}(1: 1,000)$ and goat anti-mouse IgG-HRP $(1: 1,000)$. $\mathrm{MIG} 7 / \beta$-actin ratio was used for relative expression of proteins.

MIG7 shRNA constructs. Two target sequences and one negative control (Neo) sequence were synthesized based on the sequence of human MIG7 (GenBank no. DQ080207.2) and cloned into pSIREN vector to make pSIREN-M1 (MIG7 shRNA-1), pSIREN-M2 (MIG7 shRNA-2) shRNA constructs or $M I G 7$ Neo construct pSIREN-MN (MIG7 shRNA-N) construct. The target sequences were as follows: Oligo \#1: 
Table I. Expression of MIG7 protein and vasculogenic mimicry (VM) formation in HCC tissues.

\begin{tabular}{lccccccc}
\hline & \multicolumn{3}{c}{ MIG7 } & & & VM \\
\cline { 2 - 4 } \cline { 5 - 7 } &,- 0 &,+ 1 &,++ 2 & &,- 0 &,+ 1 &,++ 2 \\
\hline HCC specimens, $\mathrm{n} /$ total $(\%)$ & $8 / 40(20)$ & $18 / 40(45)$ & $14 / 40(35)$ & & $6 / 40(15)$ & $20 / 40(50)$ & $14 / 40(35)$ \\
\hline
\end{tabular}

$\mathrm{r}_{\mathrm{s}}=0.554, \mathrm{P}<0.001$.

5'-GAT CCA AAG TTT CAT TCT TCG ACT TCA AGA GAG TCG AAG AAA TGA AAC TTT TTT TTT G-3' and 3'-GTT TCA AAG TAA GAA GCT GAA GTT CTC TCA GCT TCT TTA CTT TGA AAA AAA AAC TTA A-5'; Oligo \#2: 5'-GGA TCC CAC AGC TTG AGT GGA ATA CTT CAA GAG AGT ATT CCA CTC AAG CTG TGT TTT TTG-3' and 3'-GGT GTC GAA CTC ACC TTA TGA AGT TCT CTC ATA AGG TGA GTT CGA CAC AAA AAA CTT AAG-5'.

Transfection. MHCC-97H cells were cultured in DMEM high glucose medium supplemented with $10 \%$ FBS (from Gibco), $80 \mathrm{U} / \mathrm{ml}$ penicillin, and $100 \mu \mathrm{g} / \mathrm{ml}$ streptomycin at $37^{\circ} \mathrm{C}$ under $5 \% \mathrm{CO}_{2}$. MHCC-97H cells were transfected with $M I G 7$ shRNA constructs or control MIG7 Neo construct using Lipofectamine 2000 (Invitrogen) following the manufacturer's instructions. The stable knockdown cells were selected by $3 \mu \mathrm{g} / \mathrm{ml}$ puromycin for 2 weeks. The stable cells in which $M I G 7$ was efficiently knocked down were named as MHCC-97H-1 cells derived from MHCC-97H+pSIREN-M1 and MHCC-97H-2 cells derived from MHCC-97H+pSIREN-M2, and the stable control cell line was named as MHCC-97H Neo. The clones were characterized by semi-quantitative PCR and western blot analysis to assess the expression of MIG7 mRNA and MIG7 protein, respectively.

$3 D$ culture after transfection. Matrigel (50 $\mu \mathrm{l} / \mathrm{hole})$ was added to 6-well plates and then the cells were incubated at $37^{\circ} \mathrm{C}$ for $20 \mathrm{~min}$. The MHCC-97H cells $\left(1 \times 10^{6} / \mathrm{ml}\right)$, from the 6 groups, were maintained in DMEM supplemented with $10 \% \mathrm{FBS}$, and were then seeded onto the gels and incubated at $37^{\circ} \mathrm{C}$ in $5 \% \mathrm{CO}_{2} / 95 \%$ air. The numbers of tube-like connections were measured in a high-power field (Leica DM ILM inverted microscope; Leica Microsystems GmbH, Wetzlar, Germany).

Transwell invasion assay. Transwell assays were used to evaluate the invasion of the cells in six groups. A total of $4 \times 10^{4}$ MHCC-97H cells were seeded in the upper chamber (24-well plates, $8-\mu \mathrm{m}$ pores) coated with Matrigel (10 $\mu \mathrm{g} / \mathrm{hole})$ extracellular matrix (ECM) gel and media containing 10\% FBS was placed in the lower chamber. The chambers were incubated at $37^{\circ} \mathrm{C}$ in $5 \% \mathrm{CO}_{2}$ for $48 \mathrm{~h}$. Invasive cells at the bottom of the membrane were stained with $0.1 \%$ crystal violet and were counted under a microscopic. Five random fields were analyzed in each chamber.

Transwell migration assay. A total of $1 \times 10^{4} \mathrm{MHCC}-97 \mathrm{H}$ cells were seeded in the upper chamber (24-well plates, $8-\mu \mathrm{m}$ pores) coated with Matrigel (10 $\mu \mathrm{g} /$ hole) extracellular matrix (ECM) gel and media containing $20 \%$ FBS was placed in the lower chamber. The chambers were incubated at $37^{\circ} \mathrm{C}$ in $5 \%$ $\mathrm{CO}_{2}$ for $24 \mathrm{~h}$. Cells that migrated to the bottom of the insert were stained with $0.1 \%$ crystal violet and were counted under a microscopic (Leica DM ILM inverted microscope). Five random fields were analyzed in each chamber.

Cellular adhesion assay. MHCC-97H cells (1 ml cell suspension, $\left.1 \times 10^{6} / \mathrm{ml}\right)$ were placed in an EP tube $(1.5 \mathrm{ml})$ and incubated at $37^{\circ} \mathrm{C}$ in $5 \% \mathrm{CO}_{2}$ for $12 \mathrm{~h}$. The number of individual cells were counted at 2, 6 and $12 \mathrm{~h}$, respectively. The lower proportion of remining single cells, the stronger the cell-to-cell adhesion.

Statistical methods. SPSS ver20.0 (IBM SPSS, Armonk, NY, USA) was used for statistical analyses. Rank-sum test was used for ranked data. $\mathrm{P}<0.05$ was considered statistically significant.

\section{Results}

Positive association of MIG7 expression with VM formation in HCC tissues. MIG7 expression and VM formation in 40 matched pairs of HCC specimens from 40 patients were detected by immunostaining. The results (Table I) showed that the expression of MIG7 (Fig. 1A) was negative in $20 \%$ of the cases (8/40), weakly positive in $45 \%$ of the cases (18/40) and strongly positive in $35 \%$ of the cases (14/40). Meanwhile, VM formation (Fig. 1B) was negative in $15 \%$ of the cases $(6 / 40)$, weakly positive in $50 \%$ of the cases (20/40) and strongly positive in $35 \%$ of the cases (14/40). The results showed that there was a positive correlation between MIG7 expression and VM formation $\left(\mathrm{r}_{\mathrm{s}}=0.554 ; \mathrm{P}<0.001\right)$. There was no MIG7 expression and VM formation in normal liver tissues.

Positive correlation between VMformation and the metastatic potential of the HCC cell lines in $3 D$ culture. After $6 \mathrm{~h}$ of incubation, the difference in proliferation among the 4 types of cells (MHCC-97H, MHCC-97L, Huh-7 and L-02) was observed, and then it became more notable after 12 and $18 \mathrm{~h}$. We found that (Fig. 2) the density of VM formation in the MHCC-97H group $(\bar{x}=21.2)$ was higher than that of the MHCC-97L group $(\overline{\mathrm{x}}=6.8)(\mathrm{P}=0.000)$ and Huh-7 group $(\overline{\mathrm{x}}=1.6)(\mathrm{P}=0.000)$. VM formation in the different HCC cell lines varied, and it was coincident with the metastatic potential of the HCC cell lines. Moreover, there was no VM formation observed in the normal liver L-02 cells after 6,12 and $18 \mathrm{~h}$. These results showed that there was a positive correlation between VM formation and the metastatic potential of the HCC cell lines.

Positive correlation between MIG7 expression and the metastatic potential of the HCC cell lines. The expression of MIG7 

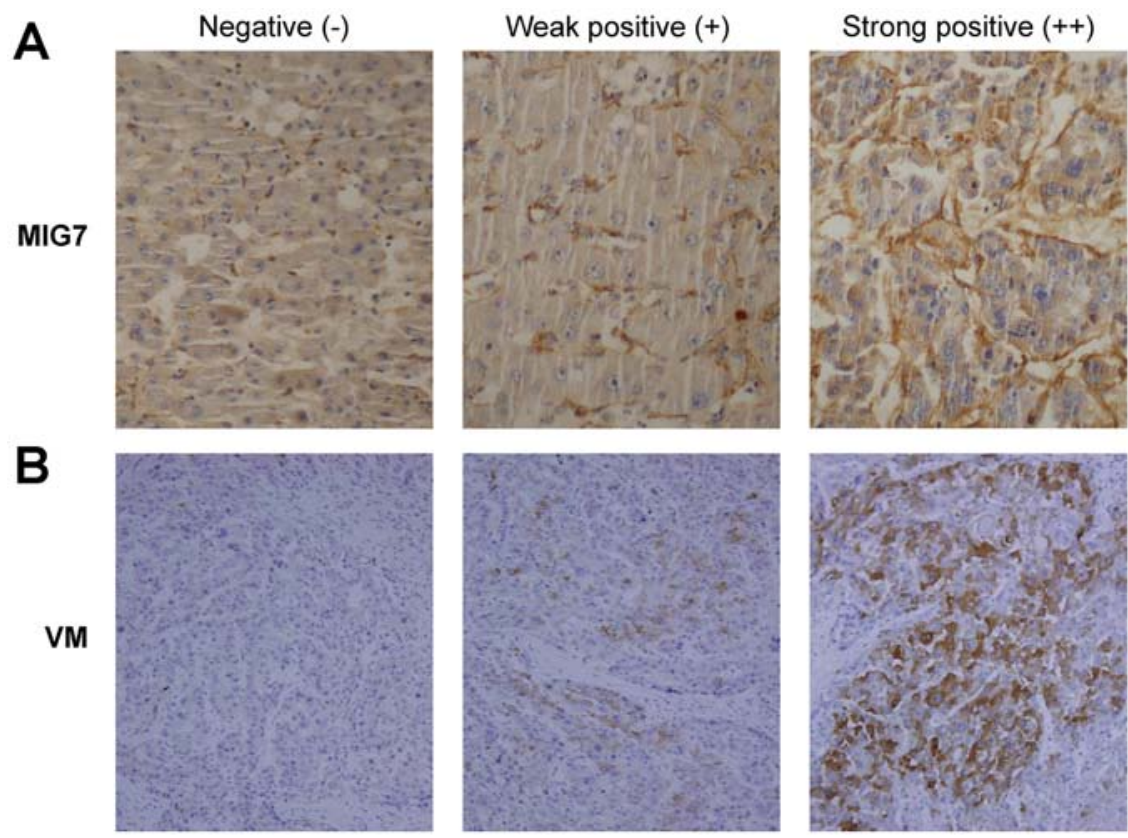

Figure 1. Immunohistochemical analysis of MIG7 expression and vasculogenic mimicry (VM) formation detection in HCC specimens. (A) The expression of MIG7 was detected by immunostaining. (B) VM formation was detected by immunostaining. The results showed that there was a positive correlation between MIG7 expression and VM formation. Magnification, x200.
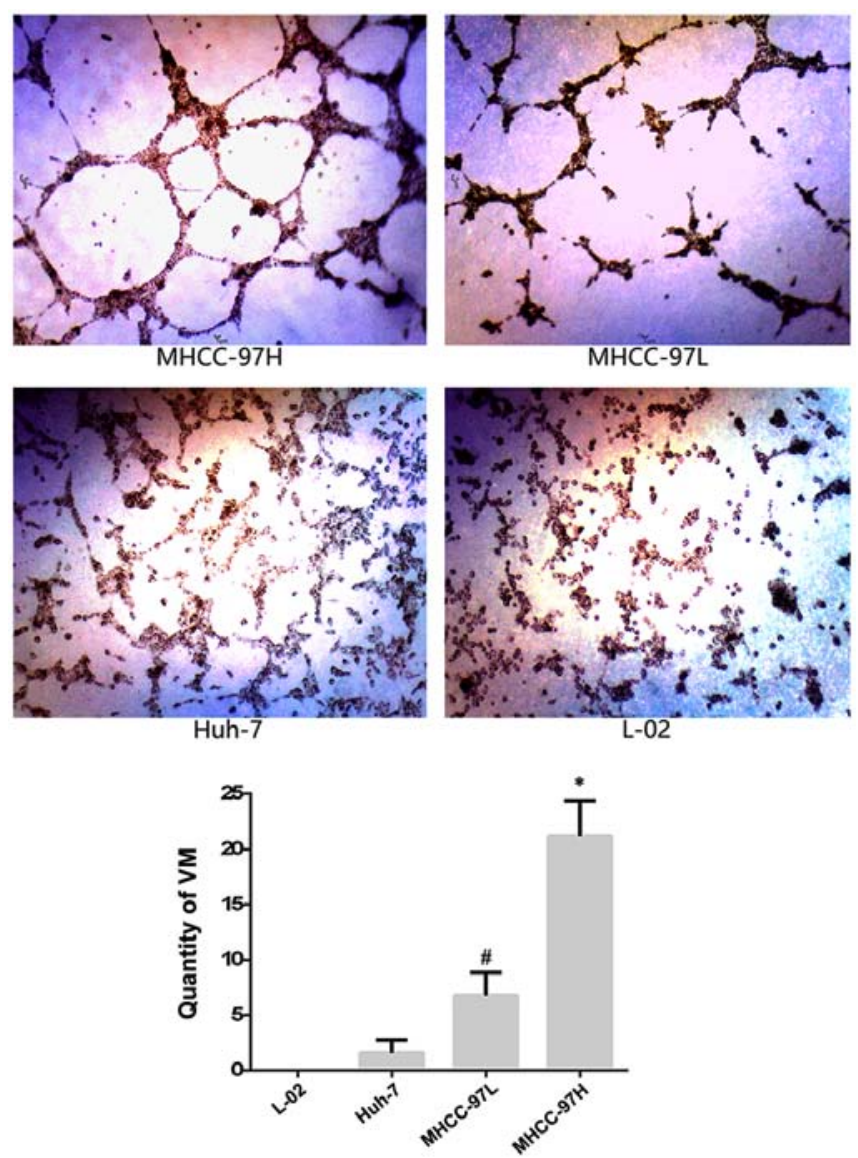

Figure 2. Vasculogenic mimicry (VM) formation was detected by 3D culture. After $18 \mathrm{~h}$ of culture of the 4 types of HCC cells (MHCC-97H, MHCC-97L, Huh-7) and a human normal hepatocyte cell line L-02, the results indicated that the density of VM in the MHCC-97H group was higher than that of the MHCC-97L group and Huh-7 group. There was no VM formation in the normal liver cell L-02 group. Scale bars, $10 \mu \mathrm{m}$. ${ }^{*} \mathrm{P}<0.01$, $(\mathrm{P}=0.000)$, compared with MHCC-97L, Huh-7 and L-02 groups; ${ }^{\prime} \mathrm{P}<0.01,(\mathrm{P}=0.000)$, compared with Huh-7. was detected in the different cell groups. The results (Fig. 3A) showed that the HCC cell lines with different metastatic potential had diverse expression of $M I G 7$ mRNA. Among them, the level of MIG7 mRNA expression in MHCC-97H cells was higher than that of MHCC-97L $(\mathrm{P}=0.000)$ and Huh-7 $(\mathrm{P}=0.000)$, and it was negative in $\mathrm{L}-02$ cells. Western blot analysis was used to detect the MIG7 protein in the 4 types of HCC cell lines. The results (Fig. 3B) indicated that the expression of MIG7 protein in MHCC-97H cells was higher than that in the MHCC-97L and Huh-7 cells. No MIG7 protein was detected in the normal liver L-02 cells.

Decreased VM formation in MHCC-97H cells with MIG7 knockdown. After $24 \mathrm{~h}$ from transfection with the constructs for knockdown, the green fluorescent protein, expressed by the constructed plasmid, was observed under a fluorescence inverted microscope and the transfection efficiency was 10-15\% (Fig. 4B). The transfected cells were selected by puromycin and green fluorescent protein labeling the positive cells increased gradually. Then, the cells were cultivated and selected by limited dilution twice. Finally, the green fluorescent protein-labeled positive cells accounted for $>90 \%$ (Fig. 4C). The knockdown efficiency of $M I G 7$ shRNA was detected by semi-quantitative PCR (Fig. 5A) and western blot analysis (Fig. 5B). The results showed that $M I G 7 \mathrm{mRNA}$ and protein was deceased significantly in both the $M I G 7$ shRNA-1 and $M I G 7$ shRNA-2 groups, especially in the MIG7 shRNA-1 group. The inhibitory efficiency of shRNA on MIG7 expression in the MIG7 shRNA-1 group was $\sim 70 \%$ compared with $M I G 7$ shRNA-N group. 3D culture was utilized to detect the inhibitory effect of $M I G 7$ shRNA on VM formation. The results (Fig. 6) showed that VM formation was significantly decreased in the MIG7 shRNA-1 ( $\overline{\mathrm{x}}=1.8)$ and MIG7 shRNA-2 groups $(\bar{x}=3.2)$ compared with the control group $(\bar{x}=26.4$; $\mathrm{P}=0.001,0.001) . M I G 7$ shRNA-N group $(\overline{\mathrm{x}}=25.8)$, empty vector 
A

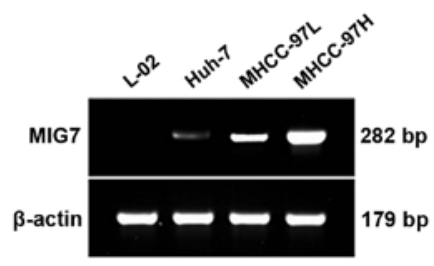

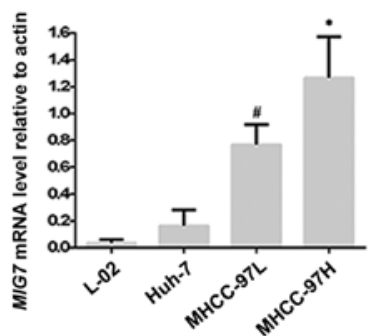

B

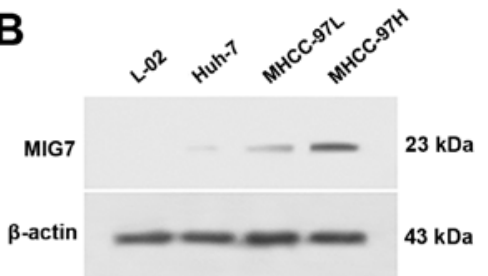

Figure 3. MIG7 mRNA and protein were detected by semi-quantitative PCR and western blot assay, respectively. (A) MIG7 mRNA was detected with semiquantitative PCR. The results showed that the expression of MIG7 mRNA in MHCC-97H cells was higher than that in the MHCC-97L, Huh-7 and L-02 cells ${ }^{*} \mathrm{P}<0.01,(\mathrm{P}=0.000)$, compared with MHCC-97L, Huh-7 and L-02 groups; $\mathrm{P}<0.01,(\mathrm{P}=0.000)$, compared with Huh-7 and L-02 groups. (B) MIG7 protein was detected by western blot assay. The results indicated that the expression of MIG7 protein in MHCC-97H cells was higher than that in the MHCC-97L and Huh-7 cells.
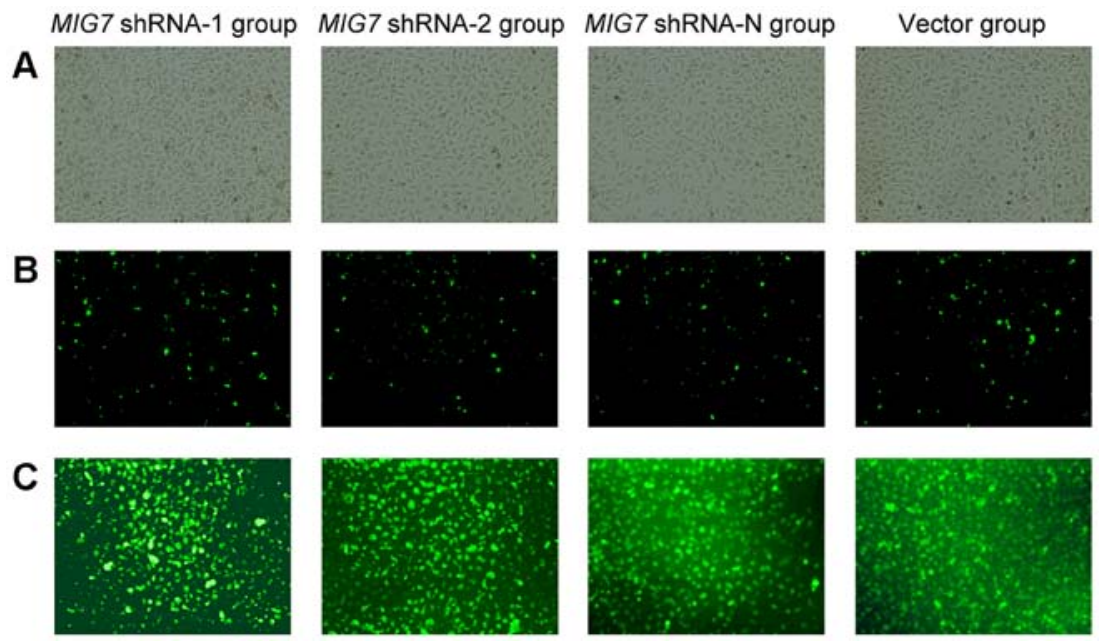

Figure 4. Transfection. (A) After $24 \mathrm{~h}$ from transfection with the constructs for knockdown, the green fluorescent protein was observed under a fluorescence inverted microscope and the transfection efficiency was $10-15 \%$ (original magnification, $x 400$ ). (B) The transfected cells were selected by puromycin, and green fluorescent protein-labeled positive cells increased gradually. (C) Finally, the green fluorescent protein-labeled positive cells accounted for $>90 \%$ (original magnification, $\mathrm{x} 400$ ).

A

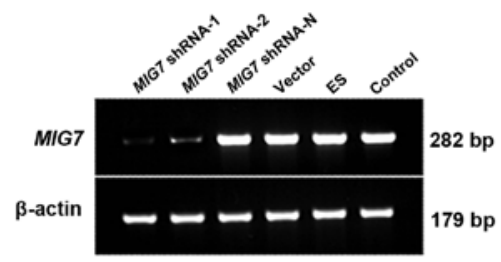

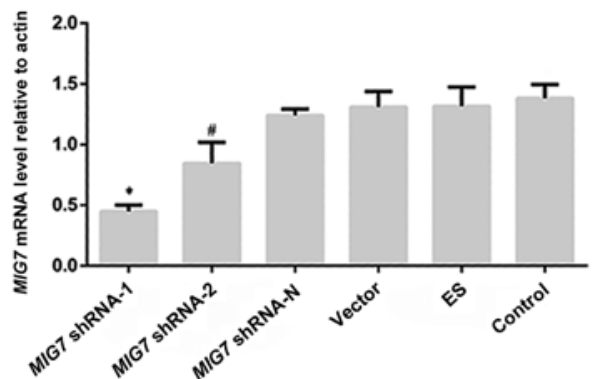

B

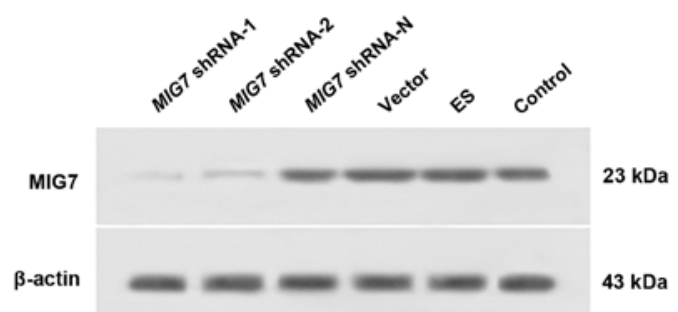

Figure 5. After transfection, MIG7 mRNA, MIG7 protein and vasculogenic mimicry (VM) formation were detected by semi-quantitative PCR, western blot assay and 3D culture, respectively. (A) Expression of MIG7 mRNA was detected with semi-quantitative PCR. ${ }^{*} \mathrm{P}<0.01$, compared with $M I G 7$ shRNA-2 $(\mathrm{P}=0.005), M I G 7$ shRNA-N $(\mathrm{P}=0.000)$, vector $(\mathrm{P}=0.000)$, $\mathrm{ES}(\mathrm{P}=0.000)$ and control groups $(\mathrm{P}=0.000) ;{ }^{*} \mathrm{P}<0.01$, compared with $M I G 7$ shRNA-N $(\mathrm{P}=0.005)$, vector $(\mathrm{P}=0.005)$, ES $(\mathrm{P}=0.007)$ and control groups $(\mathrm{P}=0.002)$. (B) The expression of MIG7 protein was detected by western blot assay. The results showed that MIG7 protein was deceased significantly in both MIG7 shRNA-1 and MIG7 shRNA-2 groups, especially in the MIG7 shRNA-1 group. 

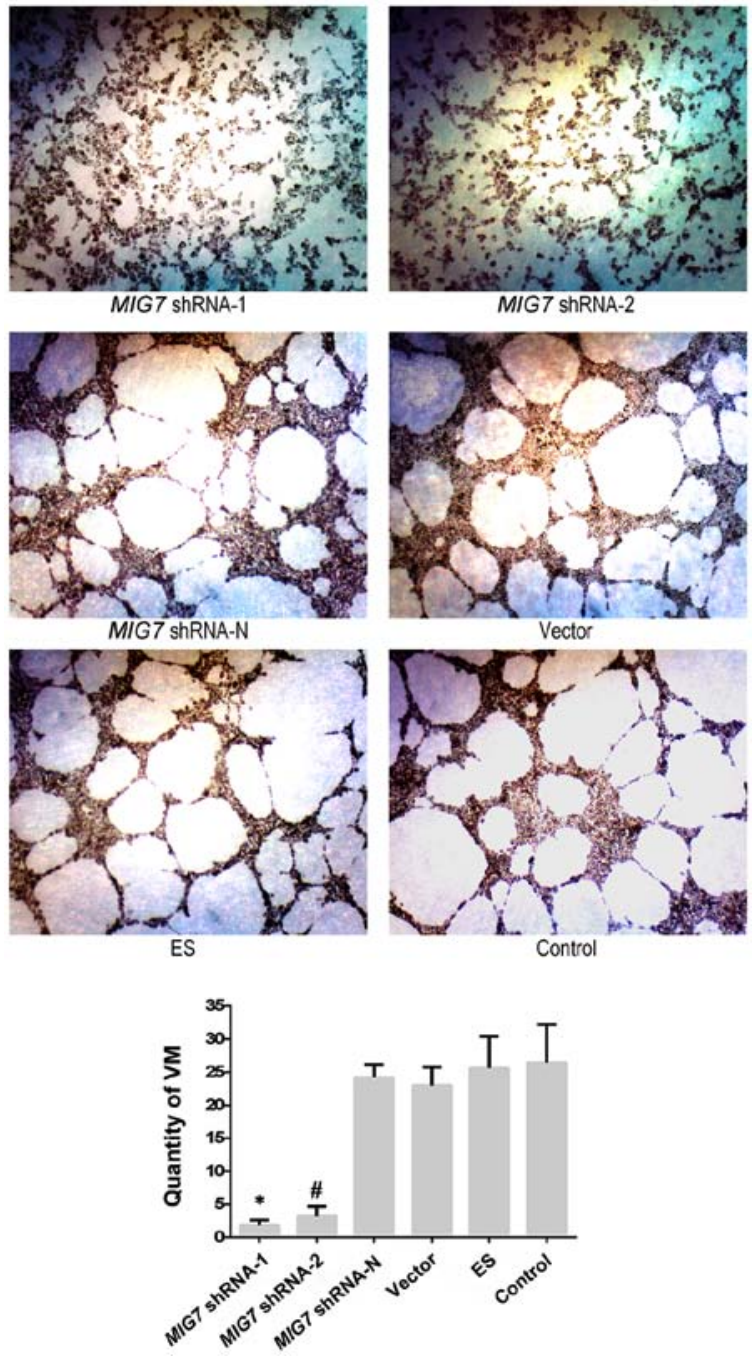

Figure 6. After transfection, the results of 3D culture showed that the quantity of VM in the $M I G 7$ shRNA-1 and $M I G 7$ shRNA-2 groups was significantly lower than that in the MIG7 shRNA-N, ES, empty vector, and the control group $(\mathrm{P}=0.000) . M I G 7$ shRNA-N group, ES group and empty vector group did not have significant difference compared with the MHCC-97H cell group $(\mathrm{P}>0.05)$ ${ }^{*} \mathrm{P}<0.01$, compared with $M I G 7$ shRNA-N, vector, ES and control groups; ${ }^{*} \mathrm{P}<0.01$, compared with $M I G 7$ shRNA-N, vector, ES and control groups.

group $(\overline{\mathrm{x}}=23)$ and ES group $(\overline{\mathrm{x}}=25.6)$ did not have a significant difference compared with the control group $(\mathrm{P}=0.842$, $\mathrm{P}=0.284$ and $\mathrm{P}=0.819$, respectively). The results indicated that $M I G 7$ plays an important role in VM formation.

Suppressed invasive properties and increased cellular adhesion in MHCC-97H cells with MIG7 knockdown. To evaluate the invasive properties of the MHCC-97H cells with MIG7 knockdown, we performed Transwell invasion assay (Fig. 7A) and Transwell migration assay (Fig. 7B). The result of the Transwell invasion assay $(48 \mathrm{~h})$ showed that the number of cells that invaded to the lower portion of the chamber in the $M I G 7$ shRNA-1 ( $\overline{\mathrm{x}}=112)$ and $M I G 7$ shRNA-2 groups $(\overline{\mathrm{x}}=146)$ was significantly lower than that of in $M I G 7$ shRNA-N group $(\overline{\mathrm{x}}=360 ; \mathrm{P}=0.000,0.000)$, empty vector group $(\overline{\mathrm{x}}=358$; $\mathrm{P}=0.000,0.000)$, ES group $(\overline{\mathrm{x}}=365.2 ; \mathrm{P}=0.000,0.000)$ and MHCC-97H cell group ( $\bar{x}=367 ; P=0.000,0.000)$. The results also indicated that there were no significant differences between the MIG7 shRNA-N group, empty vector group, ES
A
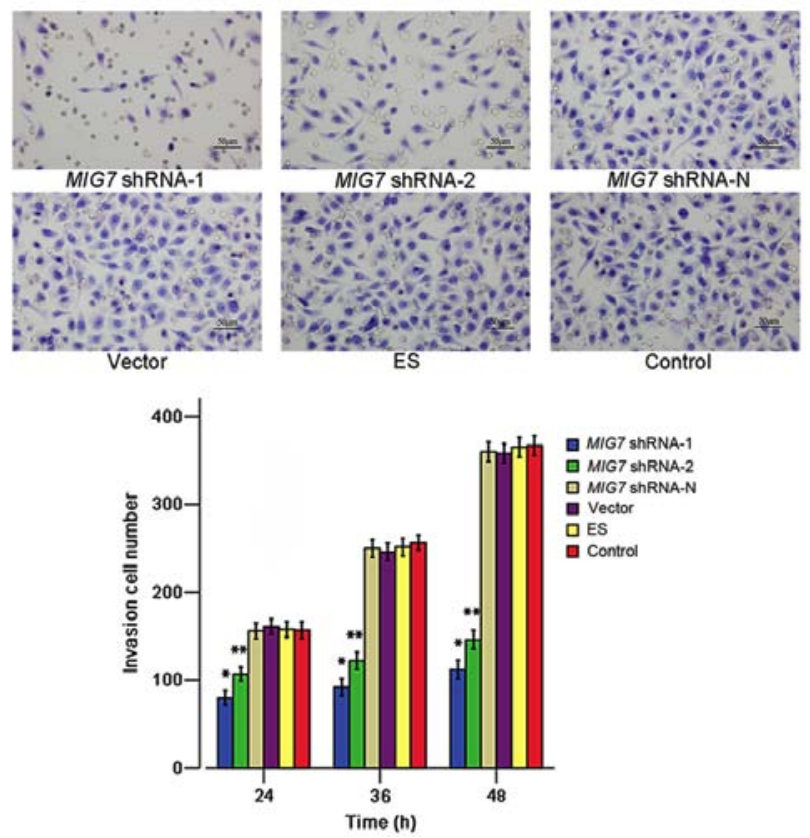

B

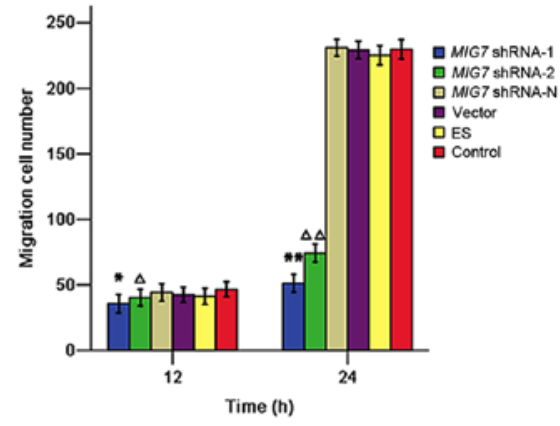

C

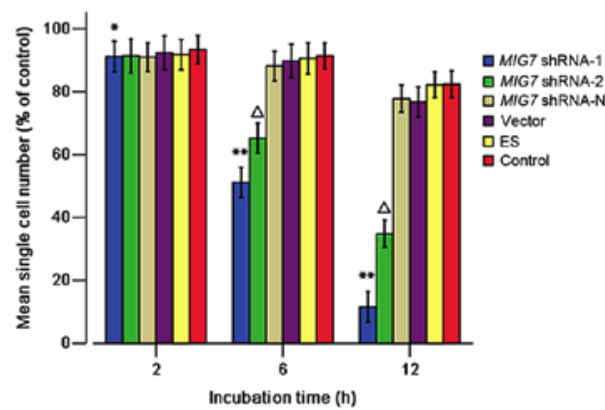

Figure 7. Transwell invasion, migration and cellular adhesion assay. (A) The Transwell invasion assay showed that the $M I G 7$ shRNA inhibited cell invasive ability. " $\mathrm{P}<0.01$, compared with $M I G 7$ shRNA-2 ( $\mathrm{P}=0.008)$, MIG7 shRNA-N $(\mathrm{P}=0.000)$, vector $(\mathrm{P}=0.000)$, $\mathrm{ES}(\mathrm{P}=0.000)$ and control groups $(\mathrm{P}=0.000)$; ${ }^{* *} \mathrm{P}<0.01$, compared with $M I G 7$ shRNA-N $(\mathrm{P}=0.000)$, vector $(\mathrm{P}=0.000)$, ES $(\mathrm{P}=0.000)$ and control groups $(\mathrm{P}=0.000)$. There was no significant difference between MIG7 shRNA-N, vector, ES and control groups $(\mathrm{P}>0.05)$. (B) The Transwell migration assay showed that the cell migration ability was suppressed by $M I G 7$ shRNA. "P $>0.05$, compared with $M I G 7$ shRNA-2, MIG7 shRNA$\mathrm{N}$, vector, ES and control groups; ${ }^{* *} \mathrm{P}<0.05$, compared with $M I G 7$ shRNA-N $(\mathrm{P}=0.031)$, vector $(\mathrm{P}=0.000)$, $\mathrm{ES}(\mathrm{P}=0.000)$ and control groups $(\mathrm{P}=0.000)$; ${ }^{\triangle} \mathrm{P}>0.05$, compared with MIG7 shRNA-N, vector, ES and control groups; ${ }^{\Delta \Delta} \mathrm{P}<0.01$, compared with MIG7 shRNA-N $(\mathrm{P}=0.000)$, vector $(\mathrm{P}=0.000)$, ES $(\mathrm{P}=0.000)$ and control groups $(\mathrm{P}=0.000)$. There was no significantly difference between MIG7 shRNA-N, vector, ES and control groups ( $\mathrm{P}>0.05)$. (C) The results of cellular adhesion assay showed that after $6 \mathrm{~h}$ from incubation, $M I G 7$ shRNA increased cellular adhesion. "P $>0.05$, compared with $M I G 7$ shRNA-2, $M I G 7$ shRNA-N, vector, ES and control groups; ${ }^{* *} \mathrm{P}<0.01$, compared with $M I G 7$ shRNA-2 ( $\mathrm{P}=0.003), M I G 7$ shRNA-N $(\mathrm{P}=0.000)$, vector $(\mathrm{P}=0.000)$, ES $(\mathrm{P}=0.000)$ and control groups $(\mathrm{P}=0.000) ;{ }^{\wedge} \mathrm{P}<0.01$, compared with $M I G 7$ shRNA-N, vector, ES and control groups. There was no significantly difference between MIG7 shRNA-N, vector, ES and control groups $(\mathrm{P}>0.05)$. 
group and MHCC-97H cell group ( $\mathrm{P}=0.549)$. The Transwell migration assay showed that the cell migration in the $M I G 7$ shRNA- $1(\overline{\mathrm{x}}=51.25)$ and $M I G 7$ shRNA- 2 groups $(\overline{\mathrm{x}}=74.25)$ was significantly lower than that of the MIG7 shRNA-N ( $\overline{\mathrm{x}}=231$; $\mathrm{P}=0.000,0.000)$, empty vector $(\overline{\mathrm{x}}=229.25 ; \mathrm{P}=0.000,0.000)$, ES $(\overline{\mathrm{x}}=225.25 ; \mathrm{P}=0.000,0.000)$ and MHCC-97H cell group $(\bar{x}=229.75 ; P=0.000,0.000)$ after $24 \mathrm{~h}$ from transfection. We also assessed the cellular adherent ability using cellular adhesion assay. The results (Fig. 7C) showed that after $6 \mathrm{~h}$ of incubation, the number of single cells in the $M I G 7$ shRNA-1 $(\overline{\mathrm{x}}=51.2)$ and $M I G 7$ shRNA-2 groups $(\overline{\mathrm{x}}=65.2)$ was significantly lower than that of in MIG7 shRNA-N group ( $\overline{\mathrm{x}}=88.2 ; \mathrm{P}=0.000$, $0.000)$, empty vector group ( $\bar{x}=89.8 ; \mathrm{P}=0.000,0.000)$, ES group $(\overline{\mathrm{x}}=90.6 ; \mathrm{P}=0.000,0.000)$ and MHCC-97H cell group $(\overline{\mathrm{x}}=91.4$; $\mathrm{P}=0.000,0.000$ ). Moreover, the number of single cells in the $M I G 7$ shRNA-1 group was significantly lower than that of the $M I G 7$ shRNA-2 group ( $\mathrm{P}=0.000)$; there were no significant differences among the $M I G 7$ shRNA-N group, empty vector group, ES group and MHCC-97H cell group $(\mathrm{P}=0.431)$. These results suggest that $M I G 7$ plays an important role in regulating HCC cellular adhesion and invasive properties.

\section{Discussion}

The metastasis of HCC is one of the major reasons responsible for the failure of HCC treatment and the death of patients. Therefore, more attention should be focused on the mechanism of HCC metastasis. In the present study, we found a positive correlation between $M I G 7$ and VM in both clinical specimens and in vitro experiments. MIG7 knockdown in 3D cultured MHCC-97H cells reduced the VM formation and weakened the invasive properties accompanied by enhanced cellular adhesion. Thus, this study provides evidence for a causal association of MIG7 with VM formation in HCC, which suggests that MIG7 could be a potential treatment target for cancer invasion and metastasis.

Previously, VM has been described in HCC and was associated with advanced tumor grade, invasion, metastasis and poor patient prognosis $(23,24)$. More studies investigated the relevant mechanisms and signaling pathways of VM formation, such as hypoxia inducible factor 1- $\alpha$ (HIF-1 $\alpha$ ), (25) matrix metalloproteinases (MMPs), phosphoinositide 3-kinase (PI3K), laminin $5(\operatorname{Ln}-5) \gamma^{2}$ chain $(26,27)$, focal adhesion kinase (FAK) (28) vascular endothelial-cadherin (VE-cadherin) (29) and epithelial cell kinase (EphA2) (30). However, the exact and detailed mechanisms underlying VM remain unclear. In the present study, we found that MIG7 and VM were highly expressed in HCC tissues and that $M I G 7$ has a significant positive correlation with VM formation. We also found that $M I G 7$ expression in different HCC cell lines was coincident with VM formation, invasion and metastasis. The result was consistent with previously reported studies which showed a positive correlation of $M I G 7$ with VM in human lung cancer and gastric carcinoma $(20,31)$.

Based on the analyses above, we proposed that MIG7 may induce the invasion and metastasis of HCC by regulating VM formation. Furthermore, the metastasis of HCC may be inhibited if the expression of $M I G 7$ is downregulated. Downregulation of $M I G 7$ may be an effective and safe therapeutic strategy. Based on the data above, the overexpression of $M I G 7$ generally leads to a poor prognosis and there is no $M I G 7$ expression and VM formation in normal liver tissues. Therefore, MIG7 can be considered as a potential safe and specific molecular target for HCC gene therapy. The invasion and metastasis of HCC could be suppressed if we transport MIG7 antagonist into HCC cells in order to inhibit VM formation. This therapeutic strategy, targeting $M I G 7$, for HCC has great application potential. Moreover, $M I G 7$-targeting therapy combined with various treatment methods at present, especially endothelial-targeting angiogenesis inhibitors (such as ES), may show better efficacy than any single use of angiogenesis inhibitors alone.

Subsequently, in order to verify the hypothesis, RNAi technique was employed to construct the recombinant retrovirus MIG7 shRNA expression vector plasmid. Additionally, because of the high expression of MIG7 in the MHCC-97H cell line, MHCC-97H cells were chosen as effector cells to explore the role of MIG7 in VM formation and HCC metastatic regulation. After the transfection of MIG7 shRNA into MHCC-97H cells, we found that $M I G 7 \mathrm{mRNA}$ and protein were significantly decreased according to semi-quantitative PCR and western blot assay. Moreover, VM formation, invasion, migration of MHCC-97H cells were inhibited significantly by 3D culture, Transwell invasion and migration assay, respectively $(\mathrm{P}<0.05)$, while the adhesion capability of MHCC-97H cells was increased significantly $(\mathrm{P}<0.05)$. However, there was no significant effect of ES on MIG7 expression and intercellular adhesion, invasion and metastasis.

A recent study reported that intra-tumor heterogeneity does exist in patients with HCC (32). The authors performed genome sequencing on 43 lesions from 10 patients with hepatitis B virus (HBV)-associated HCC and compared the genetic features of different lesions from each patient. They found that the mutations which were shared by all the lesions in each patient varied from 8 to $97 \%$, indicating the indetermination existing in intra-tumor heterogeneity. Thus, the genomic features of HCC in patients might not be characterized by sequence analysis of one single lesion, and this would be a challenge for precision medicine in patients with HCC. Therefore, more specific targets and the key genes that link to them are still needed to be identified in order to facilitate effective and specific precision medicine for tumor treatment.

In closing, $M I G 7$ expression in $\mathrm{HCC}$ tissue is correlated positively with VM formation and $M I G 7$ expression in different HCC cell lines is coincident with their VM formation, invasion and metastasis. Moreover, MIG7 shRNA inhibits $M I G 7$ expression, VM formation and HCC invasion and metastasis stably and effectively. There was no significant effect of ES on MIG7 expression, VM formation and intercellular adhesion, invasion and metastasis. However, relevant in vivo experiments are still necessary for investigation.

\section{Acknowledgements}

This study was supported by the National Natural Science Foundation of China (no. 81272547) and Tianjin Science and Technology Project (15ZXLCSY00040).

\section{References}

1. Ferlay J, Soerjomataram I, Dikshit R, Eser S, Mathers C, Rebelo M, Parkin DM, Forman D and Bray F: Cancer incidence and mortality worldwide: sources, methods and major patterns in GLOBOCAN 2012. Int J Cancer 136: E359-E386, 2015. 
2. European Association for Study of Liver; European Organisation for Research and Treatment of Cancer: EASL-EORTC clinical practice guidelines: management of hepatocellular carcinoma. Eur J Cancer 48: 599-641, 2012.

3. Folberg R, Hendrix MJC and Maniotis AJ: Vasculogenic mimicry and tumor angiogenesis. Am J Pathol 156: 361-381, 2000.

4. Chen L, He Y, Sun S, Sun B and Tang X: Vasculogenic mimicry is a major feature and novel predictor of poor prognosis in patients with orbital rhabdomyosarcoma. Oncol Lett 10: 1635-1641, 2015.

5. Yang JP, Liao YD, Mai DM, Xie P, Qiang YY, Zheng LS Wang MY, Mei Y, Meng DF, Xu L, et al: Tumor vasculogenic mimicry predicts poor prognosis in cancer patients: A metaanalysis. Angiogenesis 19: 191-200, 2016.

6. Zhao N, Sun BC, Zhao XL, Wang Y, Meng J, Che N, Dong XY and $\mathrm{Gu} \mathrm{Q}$ : Role of Bcl-2 and its associated miRNAs in vasculogenic mimicry of hepatocellular carcinoma. Int J Clin Exp Pathol 8: 15759-15768, 2015.

7. Tang J, Wang J, Fan L, Li X, Liu N, Luo W, Wang J, Wang Y and Wang Y: cRGD inhibits vasculogenic mimicry formation by down-regulating uPA expression and reducing EMT in ovarian cancer. Oncotarget 7: 24050-24062, 2016.

8. Hendrix MJ, Seftor EA, Seftor RE, Chao JT, Chien DS and Chu YW: Tumor cell vascular mimicry: Novel targeting opportunity in melanoma. Pharmacol Ther 159: 83-92, 2016.

9. Ren K, Yao N, Wang G, Tian L, Ma J, Shi X, Zhang L, Zhang J, Zhou X, Zhou G, et al: Vasculogenic mimicry: A new prognostic sign of human osteosarcoma. Hum Pathol 45: 2120-2129, 2014.

10. Luo F, Yang K, Liu RL, Meng C, Dang RF and Xu Y: Formation of vasculogenic mimicry in bone metastasis of prostate cancer: Correlation with cell apoptosis and senescence regulation pathways. Pathol Res Pract 210: 291-295, 2014.

11. Folkman J: Antiangiogenesis in cancer therapy - endostatin and its mechanisms of action. Exp Cell Res 312: 594-607, 2006.

12. Skovseth DK, Veuger MJ, Sorensen DR, De Angelis PM and Haraldsen G: Endostatin dramatically inhibits endothelial cell migration, vascular morphogenesis, and perivascular cell recruitment in vivo. Blood 105: 1044-1051, 2005.

13. Alahuhta I, Aikio M, Väyrynen O, Nurmenniemi S, Suojanen J, Teppo S, Pihlajaniemi T, Heljasvaara R, Salo T and Nyberg P: Endostatin induces proliferation of oral carcinoma cells but its effect on invasion is modified by the tumor microenvironment. Exp Cell Res 336: 130-140, 2015.

14. Qu B, Guo L, Ma J and Lv Y: Antiangiogenesis therapy might have the unintended effect of promoting tumor metastasis by increasing an alternative circulatory system. Med Hypotheses 74 360-361, 2010

15. Eikesdal HP and Kalluri R: Drug resistance associated with antiangiogenesis therapy. Semin Cancer Biol 19: 310-317, 2009.

16. Liu YR, Sun B, Zhao XL, Gu Q, Liu ZY, Dong XY, Che N and Mo J: Basal caspase-3 activity promotes migration, invasion, and vasculogenic mimicry formation of melanoma cells. Melanoma Res 23: 243-253, 2013.

17. Linder $\mathrm{M}$ and Tschernig T: Vasculogenic mimicry: Possible role of effector caspase-3, caspase-6 and caspase-7. Ann Anat 204: 114-117, 2016.

18. Petty AP, Garman KL, Winn VD, Spidel CM and Lindsey JS: Overexpression of carcinoma and embryonic cytotrophoblast cell-specific Mig-7 induces invasion and vessel-like structure formation. Am J Pathol 170: 1763-1780, 2007.
19. Feng X, Yao J, Gao X, Jing Y, Kang T, Jiang D, Jiang T, Feng J, Zhu Q, Jiang X, et al: Multi-targeting peptide-functionalized nanoparticles recognized vasculogenic mimicry, tumor neovasculature and glioma cells for enhanced anti-glioma therapy. ACS Appl Mater Interfaces 7: 27885-27899, 2015.

20. Ho MY, Liang CM and Liang SM: MIG-7 and phosphorylated prohibitin coordinately regulate lung cancer invasion/metastasis. Oncotarget 6: 381-393, 2015.

21. Li WL and Gao Q: Mig-7 enhances vasculogenic mimicry in gastric cancer cells. Xi Bao Yu Fen Zi Mian Yi Xue Za Zhi 28: 1142-1145, 2012 (In Chinese).

22. Lissitzky JC, Parriaux D, Ristorcelli E, Vérine A, Lombardo D and Verrando P: Cyclic AMP signaling as a mediator of vasculogenic mimicry in aggressive human melanoma cells in vitro. Cancer Res 69: 802-809, 2009.

23. Liu WB, Xu GL, Jia WD, Li JS, Ma JL, Chen K, Wang ZH, Ge YS, Ren WH, Yu JH, et al: Prognostic significance and mechanisms of patterned matrix vasculogenic mimicry in hepatocellular carcinoma. Med Oncol 28 (Suppl 1): S228-S238, 2011

24. Yang Z, Sun B, Zhao X, Shao B, An J, Gu Q, Wang Y, Dong X, Zhang Y and Qiu Z: Erythropoietin and erythropoietin receptor in hepatocellular carcinoma: Correlation with vasculogenic mimicry and poor prognosis. Int J Clin Exp Pathol 8: 4033-4043, 2015.

25. Li S, Meng W, Guan Z, Guo Y and Han X: The hypoxia-related signaling pathways of vasculogenic mimicry in tumor treatment. Biomed Pharmacother 80: 127-135, 2016.

26. Liu X, Wang JH, Li S, Li LL, Huang M, Zhang YH, Liu Y, Yang YT, Ding R and Ke YQ: Histone deacetylase 3 expression correlates with vasculogenic mimicry through the phosphoinositide3-kinase/ERK-MMP-laminin5 $\gamma 2$ signaling pathway. Cancer Sci 106: 857-866, 2015.

27. Lin H, Pan JC, Zhang FM, Huang B, Chen X, Zhuang JT, Wang H, Mo CQ, Wang DH and Qiu SP: Matrix metalloproteinase-9 is required for vasculogenic mimicry by clear cell renal carcinoma cells. Urol Oncol 33: 168.e9-168.e16, 2015.

28. Zang M, Zhang Y, Zhang B, Hu L, Li J, Fan Z, Wang H, Su L, Zhu Z, Li C, et al: CEACAM6 promotes tumor angiogenesis and vasculogenic mimicry in gastric cancer via FAK signaling. Biochim Biophys Acta 1852: 1020-1028, 2015.

29. Zhao N, Sun H, Sun B, Zhu D, Zhao X, Wang Y, Gu Q, Dong X, Liu F, Zhang Y, et al: miR-27a-3p suppresses tumor metastasis and VM by down-regulating VE-cadherin expression and inhibiting EMT: An essential role for Twist-1 in HCC. Sci Rep 6: 23091, 2016.

30. Vukoja V, Brandenbusch T, Tura A, Nassar K, Rohrbach DJ, Lüke M, Grisanti $S$ and Lüke J: Expression of EphA2 in metastatic and non-metastatic primary uveal melanoma. Klin Monatsbl Augenheilkd 232: 290-297, 2016 (In German).

31. Liao S and Gao Q: Expressions and clinical significance of vasculogenic mimicry and related protein Mig-7 and MMP-2 in gastric carcinoma. Xi Bao Yu Fen Zi Mian Yi Xue Za Zhi 29: 194-196, 2013 (In Chinese).

32. Xue R, Li R, Guo H, Guo L, Su Z, Ni X, Qi L, Zhang T, Li Q, Zhang Z, et al: Variable intra-tumor genomic heterogeneity of multiple lesions in patients with hepatocellular carcinoma. Gastroenterology 150: 998-1008, 2016. 